\title{
NOTAS SOBRE LOS LLAMADOS USOS TEMPORALES «DISLOCADOS» EN LA CONVERSACIÓN COLOQUIAL
}

\author{
ANTONIO BRIZ \\ Universidad de Valencia. Grupo Val.Es.Co. \\ briz@uv.es
}

\section{Resumen}

This work talks about the discursive and strategic values of the change of tenses in colloquial speech and tries to show their regularities, what means that is not anomalous.

\section{INTROIUUCCIÓN}

E. Benveniste (1965) establecía una diferencia entre:

- el tiempo físico, el paso del tiempo:

(1)

A: no yo SÉ que debería darte más tiempo $\downarrow$ del que te doy

B: pero si yo no te pido más tiempol yo lo que te pido es que estés SEGURO/ porque si no estás seguro pues/ oye $\downarrow$ si quieres lo dejamos ¿me entiendes? yo no lo quiero dejar/ yo por mí ya sabes que/ yo te quiero mucho y yo / o sea- si ya sabcs que por mí NO/ pero si tú ' ves que necesitas un tiempo/ o- yo qué sé o que no estás scguro de que me quieras $\rightarrow$ [ML.84.A.1]

- el tiempo cronológico, el tiempo de los acontecimientos y sucesos:

(2) A: eso fue en Cou $\downarrow$ tú $\downarrow$ hace un montón de años $\downarrow$ ya

[H.38.A.1]

- y el tiempo lingüístico, basado en cl tiempo cronológico, es el tiempo de los acontecimientos, si bien vistos y medidos desde un origen o centro de referencia temporal que puede o no coincidir con el momento de habla, de la enunciación, del acto de comunicación.

Al hablar de tiempo verbal, de las relaciones temporales no referimos a esta última noción del tiempo. De este modo, al describir la forma verbal fue del ejemplo anterior afirmamos, siguiendo las aproximaciones temporalistas tradicionales que se trata de un pretérito, por tanto pasado. Siguiendo aproximaciones más modernas, quizá menos temporalistas, 
decimos que se expresa una relación temporal de anterioridad o que contiene un vector de anterioridad en relación directa con respecto a un punto de origen $O$, el momento de habla (Rojo y Veiga, 1999). Comp. sobre el esquema 1:

\section{Esquema 1}

(Rojo y Veiga, 1999: págs. 2881-2882)

canté O-V(anteriuridad): ayer yo canté una canción

canto OoV(simultaneidad): hoy canto una canción

cantará $\mathrm{O}+\mathrm{V}$ (posterioridad): mañana cantaré una canción

cantaba $(\mathrm{O}-\mathrm{V})$ oV (simultaneidad en relación a momento anterior al origen)

Completemos un poco los significados temporales con otro cuadto tomado de Rojo y Veiga (1999), que reproducimos en el esquema 2:

Esquema 2

(Rojo y Veiga, 1999: pág. 2884)

Punto

de referencia

o

$(\mathrm{O}-\mathrm{V})$

$(\mathrm{O} \circ \mathrm{V})$

$(\mathrm{O}+\mathrm{V})$

$((\mathrm{O}-\mathrm{V}) \mid \mathrm{V})$

Relación temporal primaria

$-\mathrm{V}$

(anterioridad)

canté

había cantado

he cantado

habré cantado

habría cantado
$\mathrm{oV}+\mathrm{V}$

(simultaneidad)

canto

cantaba

A partir del cuadro anterior, estos autores hacen notar los vínculos entre las formas que poseen la misma relación primaria; por ejemplo, canto y cantaba tiencn en común el ser formas que expresan primariamente simultaneidad, aunque canto sobre el origen y cantaba con relación a un punto anterior al origen, etc. Sì se tienen en cuenta el punto de referencia, se observan conexiones entre formas como canto y cantaré, o entre habia cantado, cantaba y cantaría.

Pues bien, quizá ello nos ayude a entender o a justificar más tarde algunos de los llamados usos «dislocados» o «desplazados» de las formas temporales en español actual, concretamente, en la conversación coloquial, que es el objeto de esta conferencia, incluso, el porqué sólo algunas de las formas temporales (y no todas) quedan expuestas a dichos usos. 
2. EL USO DF LOS TIEMPOS Y LA COORULNADA INMEDIATA TEMPORAL DE LA CONVERSACIÓN. LOS DESPLAZAMIENTOS DEL CENTRO DFÍCTICO

La conversación es un discurso inmediato y actual o, lo que es lo mismo, se desarrolla en la coordcnada espacio-temporal aquí-ahora-ante ti. Emisión y recepción se producen in situ de manera sucesiva, por lo que el punto de origen o centro de referencia temporal es el momento de la enunciación, ya sea real o virtualmonte (como si mañana o ayer fueran hoy). Por ello no extraña encontrar en la conversación coloquial ciertos desplazamientos temporales hacia el presente.

Es evidente que el punto de origen y momento de habla en los ejemplos de (3) y (4) no coinciden realmente:

(Alguien está contando una historia)

(3) E: en esto un día estaba yo en casa/ y estaba por las tardes y por la mañana// un jueves y un viernes que no teníamos clase// suena el timbre $\uparrow / /$ y voy a abrir $¿$ no? $\mathrm{mm}$ abro la puerta $\uparrow /$ y me pregunta por una de las tías que vivía conmigo.

[L.IS.A.2]

(Uno que esperaba una invitación del otro)

(4) V: ¡calla cabrito! que te vas y no me dices ni pío' tú.

En la intervención anterior se observa cómo el punto de referencia no es el presente de los interlocutores, sino un punto en el pasado, a pesar de lo cual el hablante utiliza tiempos cuyo vector temporal es el de la simultaneidad con respecto al origen, esto es, formas de presente. Se trata del llamado Presente histórico, presente de pasado, un uso con un enfoque temporal diferente del que aparece localizado para esa forma en el sistema verbal del español (ver esquema 2 anterior). Es decir, formas OoV (de simultaneidad temporal con respecto al origen), como suena, voy, abro, pregunta, vas, dices, se emplean ahora en relación a un punto de referencia (anterioridad con respecto al origen: $\mathrm{O}-\mathrm{V}$, sonó), aunque, decididamente, con la intención de desplazar de manera más o menos virtual este centro deíctico hacia OoV (de hacer ver un pasado, por tanto un momento que no concuerda con de habla, como si fuera presente) para lograr un determinado efecto de sentido.

Este tipo de desplazamiento no ocurre con formas temporales como he cantado (OoV) $-\mathrm{V}$, ni con cantaba ni $(\mathrm{O}-\mathrm{V})$ oV, puesto que, de por sí, ya son formas que presentan simultaneidad temporal en el punto de referencia $(\mathrm{OoV})$, como en el caso del pretérito perfecto, $o$ en su relación temporal primaria $(\mathrm{oV})$, como en el caso del imperfecto.

Con el empleo de estas formas verbales del presente, dicho pasado cronológico se traslada virtualmente al momento actual, como si los acontecimientos estuvieran sucediendo o se vivieran en el ahora de la interacción. El efecto contextual que se logra con cste nuevo enfoque temporal es obvio: hacer actuales unos hechos pasados, que se saben como pasados, aproximar dichos acontecimientos al momento de habla, al Tú, un rccurso éste personalizador (Chafe, 1982), además de vivificador y dramatizador del discurso (Briz, 1998). Nótese sobre (3), una intervención propia de un discurso narrativo, que el presente histórico marca y da relievc a las acciones centrales. Y sobre (4), obsérvese que la fuerza de la recriminación aumenta con dicha actualización. 
Lo dicho anteriormente pone de relieve, por un lado, un hecho interesante ya destacado por C. Silva (p. 262 y ss), a saber, la relación entre tipo de discurso, estrategia pragmática y rasgo lingüístico (en nuestro caso, el uso de una forma verbal concreta); por otro lado, permite entender fácilmente la conclusión que sigue: el llamado presente histórico es un desplazamiento no tanto de las relacioncs temporales como del centro deíctico o punto de origen de los acontecimientos. Si un suceso anterior al momento de habla lo entiendo o quiero que se entienda como simultáneo al origen, necesariamente he de utilizar la forma propia, esto es, el presente. Luego lo dislocado, si es que algo lo está, con respecto al sistema verbal normal no es el uso de la forma verbal, sino el punto de vista, el enfoque temporal.

El cambio no es de una relación temporal a otra

$$
\text { canté }(-\mathrm{V}) \text {--> canto (oV) }
$$

sino de un punto de referencia $\mathrm{O}-\mathrm{V}$ quc se desplaza a un punto de referencia $\mathrm{OoV}$.

En el ejemplo de (5) estamos ante el llamado presente de futuro. De nuevo, la forma de presente, me caso, es utilizada para actualizar un acontecimiento (el casamiento) que no concuerda cronológicamente con el momento de habla. La forma temporal para referirse al mismo sería casaré (aunque con más frecuencia se prefiere el uso pcrifrástico: voy a casarme el mes que viene').

\section{Un $\mathrm{O}+\mathrm{V}$ aparece ahora como $\mathrm{OoV}$}

(5) No te lo vas a creer/ pero me caso al mes que viene

Entendida tal modificación como estrategia actualizadora, en concreto, como táctica que se emplea para dar mayor certeza o de hacer más real y seguro un hecho o actuación venideros, de nuevo estaremos ante una modificación del esquema temporal y no ante una simple dislocación o uso de una forma por otra; es decir, no es un desplazamiento temporal de $+\mathrm{V}$ a oO, sino un desplazamiento táctico de un esquema $\mathrm{O}+\mathrm{V}$ a otro $\mathrm{OoV}$, por lo quc necesariamente se emplea el presente.

El cambio no es

$$
\text { cantaré }(+V)->\text { canto }(o V)
$$

sino de un punto de referencia $\mathrm{O}+\mathrm{V}$ que se desplaza a un punto de referencia $\mathrm{OoV}$

\footnotetext{
1 En el castellano de Valencia el uso perifrástico se ha impuesto al del futuro absoluto (C: y en un pajar que me he comprad/ Vicente/ voy a hacer abajo una bodega; A: te va a costar un ojo de la caral to(d)o eso); esto mismo han señalado $\mathrm{M}^{a} \mathrm{C}$. Ferrer, $\mathrm{M}^{\mathrm{a}}$ (1996) para Rosario y P. Gómez Manzano para México y Madrid. Su valor no está tanto relacionado con la inmediater o grado de proximidad con el momento de habla, hecho que corre a cargo de de ciertos modificadores (sobre el ejemplo anterior: el mes que viene), como con la mayor certeza que su uso imprime a lo dicho.
} 
3. CUANDO EL TIEMPO ES LO DE MENOS. «DISLOCACIONES》 DE LOS VECTORES TEMPORALES PRIMARIOS. UNA FORMA VERBAL POR OTRA

De todos es sabido que muchas formas verbales, junto a tiempo o, incluso, antes que tiempo expresan valores modales o modalizadores. Es decir, a veces lo de menos es la exprcsión de una relación temporal; así pues, ésta deja de scr relevante y, por tanto, unas formas verbales aparecen en lugar de otras, incluso aparecen formas alternantes.

Ahora bien, este aparente baile no afecta ni a todos los vectores, ni a todas las formas del sistema verbal español. Esta es una cuestión a la que deberemos dar respuesta, así como también matizar lo de la aparente alternancia de formas en un mismo entorno sintagmático.

El futuro añade una marca de suposición en (6):

(6)

S: ya verás tú la baraja que nos sacará (ahora) éste/ toda PRINGÁA asquerosa y aceitosa $\downarrow$ ésa sí que valdrá

$\mathrm{J}$ : y se notarán las cartas

S: y se notarán las cartas

$Y$ en (7) deja en parte de ser una marca temporal para expresar esencialmentc ese valor de suposición (en el presente):

(7) Estarás pensando que soy tonto.

En (8), la probabilidad de que sea así en cste momento, domina sobre el tiempo:

(8) Ahora estarán bailando.

Pues bien, todos esos otros valores modo-temporales o estrictamente modales están vinculados a cicrtas estrategias, actitudes, presuposiciones... Van más allá de la localización de un suceso. Inchuso, dado que dicha localización se extrae del contexto, la forma vcrbal deja en ocasiones de ser pertinente en la expresión de tiempo.

\section{Un EJEMPLO SIGNIFICATIVO: LOS VALORES MODO-TEMPORALES DFI, IMPERFECTO}

En (9), (10) y (11) tienen una serie de ejemplos de usos especialcs del imperfecto de indicativo en español peninsular, que muestra la ampliación de valores y usos de esta forma verbal, sin duda, relacionađa con su su débil posición temporal, lo que la hace apta para la subjetivización, la modalización, como ha señalado M.L. Gutiérrez, p. 178. No en vano el imperfecto es muchas veces marca de presencia del hablante.

Como veremos presentan valores de uso diferenciados.

(9)

a. La reunión era (es) para las cuatro ¿no?

b. ¡Qué chof! ¡pern si eras tú!

c. ¿Qué te creias (crees) que yo me chupaba (chupo) el dedo? 
(10)

a. De esta paella me comía yo la mitad

b. De buena gana me iba mañana

c. Si tuviera dinero, me compraba una moto

d. Me llamaba eso a mi y

e. Yo que tú me iba

f. Con tal que quisieras, lo hacias

g. En cuanto me lo pidieras, te lo daba

(11)

a. Veníamos a pedirle un favor

b. Vale que Vosotros erais policías y nosotros ladrones

c. Imagínate que ahora se nos perdía la llave

\subsection{Imperfecto en un contexto de presente, referenciado a un discurso anterior pre- supuesto}

En el caso de los ejemplos de (9) se trata de imperfectos en un contexto de presente, aunque referidos a un discurso anterior presupuesto:

(9)

a. La reunión era (es) para las cuatro ¿no?

b. ¡Qué chof! ipero si eras tú!

c. ¿Qué te crefas (crees) que yo me chupaba (chupo) el dedo?

Esta combinación del contexto de presente y de un contexto presupuesto de pasado da cucnta del porqué el imperfecto es una forma verbal adecuada para la expresión de esta relación modo-temporal (recuérdese, sobre cl esquema de 2), que el imperfecto se caracteriza. por ser temporalmente simultáneo a un punto de referencia anterior al origen: $(\mathrm{O}-\mathrm{V}) \mathrm{Oo}$, si bien aquí dicha referencia a algo anterior está presupuesta.

¿Cuál es el valor modal que tales usos añaden?:

- De incertidumbre:

(9a) La reunión era (es) para las cuatro ¿no?

(presupuesto anterior: alguien me ha dicho que hay reunión, pero no estoy seguro)

- De sorpresa (de aprobación o de desaprobación):

(9b) ¡qué chof! ipero si eras tú!

(9c) ¿Qué te creías (crees) que yo me chupaba (chupo) el dedo?

(presupuesto anterior: esperaba alguien y resulta que en su lugar vicnes tú; me considerabas ingenuo, y ahora te he demostrado que no lo soy tanto) 


\subsection{Imperfecto en un contexto de futuro (cantaba en vez de cantaria)}

Los ejemplos de (10) son imperfectos en un contexto de futuro:

a. De esta paella me comía yo la mitad

b. De buena gana me iba mañana

c. Si tuvicra dinero, me compraba una moto

d. Me llamaba eso a mí y

e. Yo que tú me iba

f. Con tal que quisieras, lo hacias

g. En cuanto me lo pidieras, te lo daba

- Para expresar un deseo:

(I0a) De esta paella me comía yo la mitad

(IOb) De buena gana me iba mañana

Mientras en los casos anteriores de (9) todavía puede observarse la relación de uso del imperfecto con su valor temporal primario, en estos nuevos casos de (10a) y (10b) dicha relación ya no sc percibc, pues lo que expresa la forma verbal es estrictamente un valor modal.

No obstante, el empleo del imperfecto parccc un uso desplazado de lo que sería formalmente más ajustado para expresar un valor modal de deseo, de algo que no ha sucedido, pero que querría que sucediera, es decir, el condicional: de +V (comería) --> Vo (comía). Volveremos más tarde sobre este hecho.

Nótese que tanto en (10a) como en (10b) hay una negación implicita «no me la comeré)» y «no me iré»».

- Con valor de futuro (en construcciones con sentido condicional: «suponiendo que tuviera dinero», «que me llamara a mí eso», «que yo fuera tú», etc.):

(10c) Si tuviera dinero, me compraba una moto

(10d) Me llamaba eso a mi y

(10e) Yo que tú me iba

(10f) Con tal que quisieras, lo hacias

(10g) En cuanto me lo pidicras, tc lo daba

A diferencia de (10a) y (10b), los imperfectos de (10c - 10g) con valor de futuro están referenciados a un presupuesto antcrior, de un no-suceso. De hecho, de todos se parte de una negación: «No he tenido dinero hasta ahora» «No me lo ha llamado hasta ahora» «No te/me he ido hasta ahora» «no he querido hasta ahora», «no me lo has pedido hasta ahora»

\section{IMPERFECTO O CONDICIONAL FN EI MISMO ENTORNO SINTAGMÁTICO}

Muchos de los ejemplos anteriores de (9) y (10) apuntan a un uso aparentemente indistinto de dos formas verbales (cantaba/cantaria), una de indicativo y otra de subjuntivo: 
Veamos el caso concreto de los ejemplos siguientes:

Si tuviera dinero, me compraría/compraba una moto Yo tendría/tenía que haber venido

Y digo aparente uso indistinto porque, aunque se trata de hechos que probablemente no sucederán o realmente no han sucedido, hay una diferencia de grado ya sea en cuanto a esta probabilidad o al grado de obligación que otorga el hablante a lo dicho.

La supuesta «neutralización» temporal y modal se produce, en todo caso, en el plano formal y scmántico, pcro no en el pragmático. Con el uso del imperfecto compraba el hecho se siente más real, realizable, probable. Del mismo modo, el grado de obligación o de imposición de una obligación aumenta con el imperfecto tenia (claro que el hablante, confesando la culpa de no haber venido, sin atenuantes, csto cs, con tenía en lugar de tendria, intenta exculparse).

Y que sea el imperfecto y no otro tiempo puede causar cierta sorpresa, aunque no tanta, pues ambos comparten el vector (O-V): «no he tenido dinero hasta ahora»; «no he venido»

Insistimos, pues, en que ambas formas no expresan el mismo contenido modal, además, por supuesto, de cxistir una variación de uso en cuanto al registro: más coloquial en el caso del imperfecto, más formal en el del condicional.

\section{6. ¿DESPlazamiento O Dislocación al AZAR?}

Hemos observado hasta aquí que entre los casos de «dislocaciones» presentados los hay que propiamente afectan al punto de referencia y otros a los vectores temporales, y que en tales casos se añade sistemáticamente un valor modal concreto (ver Rojo y Veiga, 1999: pág. 2896).

Siguiendo a estos autores, por ejemplo, en los usos ya citados del futuro por el presente: ahora estarán bailando, estarás pensando que soy tonto (comp también serán las doce), se observa que el vector de posterioridad es sustituido por otro de simultaneidad, añadiendo un valor modal de incertidumbre o suposición en el presente.

Las conexiones entre canto y cantaré, o entre cantaba y cantaria (ver de nuevo el esquema 2) en cuanto al punto de referencia (el presente y el futuro coinciden en que su valor temporal se establece directamente sobre un punto de origen; e imperfecto y condicional coinciden en que su rclación temporal se mide en relación a punto anterior al origen), nos permiten justificar tales usos «dislocados». Lo que queremos decir con lo anterior es que estos cambios no son aleatorios, que, de acuerdo con Rojo y Veiga (1999: pág. 2896), «no todas las formas verbales de español actual admiten la posibilidad de experimentar una dislocación». Esto es, tienen una razón dentro del sistema.

Que formas de futuro, con vector temporal de posterioridad, al dejar dicha marca temporal pasen a tener un valor de incertidumbre, está en consonancia con el valor de probabilidad que toda forma de futuro expresa. Y que sea precisamente el futuro y no otro tiempo el que exprese probabilidad en el presente tampoco cxtraña, dado que con el presente tiene en común el punto de origen.

Notábamos en varios ejemplos la extensión de valores del imperfecto de indicativo, así como que a dicho usos temporales se añadía un valor modal: recordemos uno de los 
ejemplos en el que el imperfecto, con vector temporal de anterioridad, se utiliza en lugar del presente, expresando una relación temporal de simultaneidad: de buena gana me comía esta paella ahora.

Que sea el imperfecto el que se utilice está justificado por el hecho de que, precisamente, comparte con la forma de presente una relación temporal primaria de simultaneidad.

El valor modal que aporta en este caso es de irrealidad, con una diferencia de grado (menor) con respecto al empleo del condicional (comería). Con aquél se logra transmitir una mayor responsabilidad de lo dicho o hecho, una mayor certeza, una mayor implicación del yo.

\section{OTROS CONTENIDOS MODALES DEL IMPERFECTO}

Se recogen aquí otros contenidos modales del imperfecto, usado en un contexto de presente: el llamado imperfecto de cortesía y el de ficción o fantasia, recogidos en (11)

(11)

a. Veníamos a pedirle un favor

b. Vale que Vosotros erais policías y nosotros ladrones

Imagínate que ahora se nos perdía la llave

\subsection{El imperfecto de cortesía}

Algunas formas verbales se especializan como recursos de expresión cortés. Asi, en (12), el condicional, el futuro y el imperfecto de indicativo o subjuntivo aparecen entre los recursos de atenuación, en tanto minimizadores de lo dicho y el decir, formas de distanciarse o de alejarse estratégicamente de lo dicho o hecho (Briz, 1998):

Me gustaría (gusta) que vinieras

Yo lo haría (hago) de otro modo

¿Tú tendrás (tienes) tabaco rubio/ por ahí por casualidad? (pelición indirecta)

Quería/Quisiera/Querría (quiero) que vinieras a mi cumpleaños

Es tal el grado de gramaticalización en la expresión de este valor modal, que la alternancia de formas, incluida la llamada «recta» (cl presente) no cs más que cl resultado de aplicar distintos grados o escalas de atenuación según la mayor o menor intensidad obligativa hacia el interlocutor o las posibilidades de aceptación que a juicio del hablante pueda tener su cnunciado ante el oycnte (nótese cn el último de los ejemplos la alternancia entre imperfecto de indicativo, subjuntivo y condicional en virtud del grado de minimización de la petición, frente a la escasa o menor atenuación de la petición con el uso del presente)

El empleo del imperfecto o del condicional de cortesía resta fuerza ilocutiva al performativo volitivo querer, y, así pues, expresa mayor distancia interpersonal, como ya señalaba E. Alarcos (1978: pág. 107): 
(a) Queria que vinieras a mi fiesta. (atenuado)

(b) Quisiera-querria que vinieras a mi fiesta ( + atenuado)

(c) Quiero que vengas a mi fiesta. (- atenuado)

\subsection{El imperfecto de ficción o fantasía (prelúdico o lúdico)}

A diferencia del anterior, su uso no es compartido por el condicional, a pesar del componente de irrealidad (ficción o fantasía) que conlleva, quizá, porque el grado de especialización funcional y de gramaticalización es mayor.

Vale que vosotros erais policías y nosotros ladrones

Imaginate que ahora se nos perdía la llave

\section{Conclusiones}

Lo anterior no ha sido sino una reflexión sobre las aportaciones que ya realizaron autores como Rojo y Veiga (1999) y otros sobre algunos usos «dislocados» de los tiempos en el español actual.

Las características propias de la conversación coloquial, en tanto hecho lingüístico-discursivo, su inmediatez, su dinamismo y carácter actual, y en tanto hecho social, las relaciones interpersonales, ayudan a entender el resultado de ciertas «dislocaciones» temporales de algunas formas verbales en tanto que expresan un valor temporal distinto del que se entiende como primario o recto. En este género discursivo son frecuentes los usos desplazados dirigidos hacia la simultaneidad con respecto al origen, ya sea desde la posterioridad, ya desde la anterioridad. Unos, como se ha notado, afectan al punto de referencia, otros al vector temporal primario.

Y siempre están al servicio de una estrategia, ya sea vinculada a la actualización del discurso, ya a la expresión del punto de vista, añadiendo en este caso un contenido modal concreto y preciso.

Todos estos «usos dislocados» son mecanismos que, como señalaban Rojo y Veiga, al interrelacionar tiempo y modo son muy económicos para el sistema. Si no fuera por estos tendrían que multiplicarse las formas de expresión temporal. Por ejemplo, el español tendria que haber incorporado nuevas formas para expresar todo este conjunto de matices (certeza, rcalidad, objetividad, evidencialidad, probabilidad, cortesía); por ejemplo, una forma para expresar la probabilidad en el presentc, otra para la expresión de la probabilidad en el pasado, otra para indicar mayor intensidad obligativa al oyente, para indicar mayor cortesía, para expresar mayor certcza, etc. O tendría que haber recurrido a otros recursos marcadores de estos efectos de sentido, por ejemplo, a ciertos sufijos, como sucede en otras lenguas e, incluso, puede documentarse en el español del área andina ${ }^{2}$.

Es decir, todos esos otros valores (anormales) están vinculados a ciertas estrategias, actitudes, presuposiciones, etc. Por tanto, la elección de una forma temporal y modal en-

2 Según G. de Granda (Ponencia en el II Simposio de análisis del discurso orat, Valencia, 1996), en el español del área andina, por influcncia del quechua, se recurre a sufjos oracionales, validadores, para marcar algunos hechos de origen y verdad (la tierra co es redonda: (cyo no puedo asegurarlo, dicen que es redonda»), los cuales, en ocasiones, han desplazado incluso los valores temporales y aspectuales. 
tre varias opciones supone siempre un efecto pragmático, así pues, podremos aceptar la existencia de la neutralización sintáctica y semántica, pero no pragmática. Además, si tales movimientos presentan un alto grado de sistematicidad tanto en las formas de expresión verbal que se mueven como en el plano estratégico, erróneo sería seguir hablando de «usos dislocados», ni siquiera desde el punto de vista gramatical. Serían usos del sistema temporal-modal del español, sin más.

\section{REFERENCIAS BIBLIOGRÁFICAS}

Alarcos, E. (1978): Estudios de Gramática Funcional del español. Madrid, Gredos, 1978.

Benveniste, E. (1965): «E1 lenguaje y la experiencia humana». En Problemas del lenguaje. Buenos Aires, págs. 3-12.

Briz. A. (1998): El español coloquial en la conversación. Esbozo de pragmagramática. Barcelona, Ariel.

Chafe, W. (1982): «Integration and Involvement in Spcaking, Writing, and Oral Literature». En D. Tannnen (ed.): Spoken and Written Language: Exploring Orality and Literacy. Norwood, N.J., Ablex, págs. 35-53.

Ferrer, $M^{a}$ C. (1996): «Características mosfosintácticas y léxicas del español hablado en Rosario». En T. Kotsch, W. Oesterreicher y K. Zimmermann (eds.): El español hablado y la cultura oral en España e Hispanoctmérica, Bibliotheca Ibero-Amcricana. Frankfurt am Main, Vcrvucrt Verlag, págs. 303-313.

Gómez Manzano, P. (1981): «Futuro absoluto en Madrid y Mćxico», cn Anuario de Letras, 26, 8485.

Gutiérrez, $\mathrm{M}^{\mathrm{a}}$ L. (1996): «Relevancia del discurso en el uso del imperfecto», REL, 26, 2, págs. $327-$ 336.

Lamíquiz, V. (1985): «El sistema verbal idealizado y su comportamiento discursivo». En Sociolinguiistica andaluza 3: El discurso sociolingüistico. Universidad de Scvilla, págs. 105-120.

Rojo, G. y Veiga, A. (1999): «El tiempo verbal. Loa ticmpos simples». En I. Bosquc y V. Demontc (eds.): Gramática descriptiva de la lengua española 2. Madrid, Espasa, págs. 2867-2934.

Silva, C. (1996): «Estratcgias sintácticas del español hablado». En . Kotsch, W. Oesterreicher y K. Zimmermann (cds.): El español hablado y la cultura oral en España e Hispanoamérica, Bibliotheca Ibero-Amcricana. Frankfurt am Main, Vervuert Verlag, págs. 261-277. 